\section{Lungenembolie: Thrombolyse bringt keine Langzeitvorteile}

Konstantinides SV et al. Impact of thrombolytic therapy on the long-term outcome of intermediate-risk pulmonary embolism. J Am Coll Cardiol 2017; 69: 1536 - 1544

Es ist eine verbreitete Vorstellung, dass durch die medikamentöse Thrombolyse einer akuten Lungenembolie negative Langzeitfolgen, wie eine persistierende pulmonalarterielle Hypertonie bzw. Rechtsherzbelastung, reduziert werden können. Eine aktuelle prospektive randomisierte Studie zeigte jetzt aber, dass sich die Langzeitergebnisse von akuten Lungenembolien nach Thrombolyse nicht von denen mit alleiniger Antikoagulationsbehandlung unterscheiden.

In die multizentrische, randomisierte, placebokontrollierte, doppelt-verblindete PEITHO-Studie (PEITHO: Pulmonary Embolism Thrombolysis) wurden zwischen 2007 und 2012 insgesamt 1006 Patienten mit akuter Lungenembolie und Zeichen der rechtsventrikulären Dysfunktion sowie erhöhten Troponinwerten als Ausdruck einer myokardialen Schädigung aufgenommen. Es erfolgte eine $1: 1$-Randomisierung in eine Thrombolysegruppe und eine Vergleichsgruppe. Die Thrombolyse wurde als einmalige Bolusgabe von Tenecteplase durchgeführt und in beiden Studiengruppen wurde über einen Zeitraum von mindestens 48 Stunden unfraktioniertes Heparin appliziert. Die nachfolgende Antikoagulanzienbehandlung erfolgte nach der gängigen Praxis der jeweiligen Zentren.

Die erstmals 2014 publizierten Kurzzeitergebnisse der PEITHO-Studie hatten eine Reduktion des kombinierten primären Endpunktes aus Versterben bzw. hämodynamischer Dekompensation innerhalb der ersten 7 Tage nach der Lungenembolie mit thrombolytischer Therapie gezeigt. Auf der anderen Seite war aber eine erhöhte Rate hämorrhagischer Schlaganfälle (2\%) und schwerwiegender extrakranieller Blutungskomplikationen $(6,3 \%)$ verzeichnet worden.

\section{Gesamtmortalität auf län- gere Sicht in beiden Studien- gruppen vergleichbar}

Im Rahmen der aktuellen Analyse wurden die Langzeitdaten von 353 Patienten im Thrombolysearm der Studie und 343 Patienten der Vergleichsgruppe ausgewertet. Die Gesamtmortalität lag innerhalb der medianen Nachbeobachtungszeit von 37,8 Monaten bei knapp 20\%; statistisch signifikante Unterschiede zwischen den beiden Studiengruppen fanden sich nicht. Das Ergebnis erkläre sich aus der Beobachtung, dass die große Mehrzahl der Todesfälle jenseits von 30 Tagen nach dem Akutereignis bestehenden Komorbiditäten und nicht etwa einem Rechtsherzversagen geschuldet sind, so die Autoren.

\section{Rechtsherzinsuffizienz- und CTEPH-Rate nach Thrombo- lyse nicht verringert}

Eine klinische Verlaufsuntersuchung 24 Monate nach der akuten Lungenembolie erfolgte bei 175 bzw. 183 Patienten der Thrombolysegruppe bzw. der Vergleichsgruppe. Jeweils ca. ein Drittel der Patienten berichteten zu diesem Zeitpunkt über persistierende Symptome, zumeist aber nur in Form einer milden Belastungsdyspnoe. Nur ca. jeder 10. Patient musste der NYHA-Klasse III oder IV zugeordnet werden. Echokardiografisch konnten keine signifikanten Unterschiede zwischen den beiden Studiengruppen im Hinblick auf eine etwaige pulmonalarterielle Hypertonie bzw. eine rechtsventrikuläre Dysfunktion festgestellt werden. Die endgültige Diagnose einer chronisch-embolischen pulmonalarteriellen Hypertonie (CTEPH) konnte bei 2,1\% der Patienten der Thrombolysegruppe und 3,2\% der Vergleichsgruppe gestellt werden, der Unterschied war statistisch nicht signifikant.
FAZIT

Die vorliegenden Daten stützen die aktuellen Leitlinienempfehlungen, die eine Thrombolysetherapie bei Patienten mit akuter Lungenembolie nur dann vorsehen, wenn es zu einer hämodynamischen Dekompensation kommt. Eine thrombolytische Therapie in der Akutsituation führte bei Patienten mit intermediärem bis hohem Risiko im Vergleich zur alleinigen Antikoagulation zu keiner längerfristigen Prognoseverbesserung und war auch nicht mit einer Verringerung klinischer Symptome vergesellschaftet.

Dr. Katharina Franke, Darmstadt 